\title{
The Art of Neonatology, the Art of High Reliability as a Response to COVID-19
}

Daved van Stralen, MD, FAAP, Thomas A. Mercer, RAdm, USN

\section{Medicine / Neonatology as Art}

Historically, art is the skill in scholarship gained by learning or practice, evident today as a Bachelor of Arts degree or study in the liberal arts. By the $17^{\text {th }}$ century, art became associated with painting and sculpture. Art, in this usage, reflects the creativity and aesthetic intention (1). Neonatology, as a specialty in medicine, "is an art, based to an increasing extent on the medical sciences, but comprising much that still remains outside the realm of any science" (2). Because both uses of the term "art" have a common root, we find the "art as creativity and aesthetic intention" can inform the "art as practice" of neonatology.

\section{"Because both uses of the term "art" have a common root, we find the "art as creativity and aesthetic intention" can inform the "art as practice" of neonatology."}

As aesthetic intention within the affective domain, art is both a part of the world and apart from the world. Independent of Knowledge, art produces feelings, which constitutes art's importance (3). This affective quality of art drives actions that Knowledge alone cannot, capable of generating an aesthetic experience similar to John Dewey's description of the experience: complete, unified experiences of how things appear to us that are controlled by the things (4).

For Dewey, aesthetics, as the combination of mind, emotions, and judgments, begins with what interests the individual. The resulting absorption creates artistic engagement in the activity, whether as a mechanic or as a physician in practice. Lack of such engagement encourages low-quality work (5). The affective drive to experience artistic engagement occurs at the level of the individual who seeks Knowledge through experience but also acquires Knowledge from others. James Spradley (6) describes culture as the "acquired knowledge people use to interpret experience and generate behavior." Learning to engage "the particular" under the guidance of elders for the greater good not only produces "practical wisdom," described by Aristotle (7), but develops the culture ethic for the "art of practice." For Spradley (6), rather than answering the questions asked by executives or outsiders, we learn more about a culture from how the individual uses Knowledge and what interests the individual.

What does a person need to know to feel the aesthetic drive for artistic engagement? We must acquire Knowledge for the art of practice, but it is aesthetic engagement that generates our professional behavior. Then, a culture of the practice is how acquired Knowledge guides our interpretation of experience, helping us develop the cultural aesthetic drive that generates artistic engagement behaviors. The study of culture in this manner comes from the participants' interests, particularly the hidden voices, rather than the interest of executives from the dominant account (6) (8). What drives artistic engagement will reveal more about culture change than following the executive's interests.

Science, the systematic Knowledge of truth and facts often understood as objective and dispassionate, primarily organizes Knowledge for understanding and prediction. As its original structure, art consists of the Knowledge obtained through experience and is, therefore, contextual, subjective, and organized for practical use. The normative, decontextualized structure of the science of medicine risks an impersonal relationship with the patient. The pragmatic and contextual nature of the art of medicine, on the other hand, engenders personal relationships. As (2) Peabody stated for the physician, failure to develop "the intimate personal relationship between physician and patient accounts for much of his ineffectiveness in the care of patients."

Contextualization of the patient creates a clinical picture, described by Peabody (2) as "not just a photograph of a man sick in bed; it is an impressionistic painting of the patient surrounded by his home, his work, his relations, his friends, his joys, sorrows, hopes, and fears." Here, we share Peabody's embodiment of the creative, aesthetic arts into the Art of Neonatology as practice, discussed later in this article.

NEONATOLOGY TODAY is interested in publishing manuscripts from Neonatologists, Fellows, NNPs and those involved in caring for neonates on case studies, research results, hospital news, meeting announcements, and other pertinent topics. Please submit your manuscript to: LomaLindaPublishingCompany@gmail.com 
Reliability in medical care, particularly during the uncertainty due to COVID-19, is more assured when practical aesthetic engagement dominates, that is, when the practice of medical care adjusts to the context and flux of the patient's circumstances. As a subjective contextualized pragmatic stance for medical practice, the art of medicine aligns with affective judgment and practical rationality (9). The art of medicine becomes more than a metaphor.

\section{"As a subjective contextualized pragmatic stance for medical practice, the art of medicine aligns with affective judgment and practical rationality (9). The art of medicine becomes more than a metaphor."}

We differentiate the affective processes that incorporate induction as "learning through observation" from cognition that relies on deduction to "confirm through observation." Judgment, unfortunately, has developed a poor reputation. We do not always trust others' judgment, and our own judgment can fool us because we use our judgment to judge our judgment. Tim Watson, a pediatrician and former K-12 educator advocates discernment as the cognitive skill distinguishing between categories. Through the art of medicine, the physician then places contextual value on the information identified; judgment becomes an effective process.

"And he shows me carefully, the valley where the two mountains of reason and emotion meet and twine their efforts together in winding streams that quietly defy your logic" (10). Vivienne, the author of that description, vividly describes the blending of facts and feelings generated when we encounter a threat within a complex situation. As medical scientists, we prefer to believe that scientific logic will protect us from false reasoning. However, even mild stress from the novelty, uncertainty, or uncontrollability of a complex situation impairs our brain's executive functions $(11,12)$. Threat provokes fear, the emotion that drives us to maintain a safe distance by taking offensive or defensive actions $(11,13)$. We experience these evolving events as a series of short intervals or static snapshots. What we observe and what we can describe quietly eludes and defies our logic.

This does not mean we diminish the objective, normative science of medicine nor do we strongly focus on the subjective, pragmatic practice of medicine. Reliability in medical care is more assured when practical engagement dominates as practice adjusts to the flux of the patient's circumstances (9). "The practice of medicine in its broadest sense includes the whole relationship of the physician with his patient. It is an art" (2). As a subjective, contextualized pragmatic stance for medical practice, the art of medicine aligns with affective judgment and practical rationality (9). The art of medicine is more than a metaphor.

In Nicomachean Ethics, Aristotle (7) described five virtues of thought: technê, epistêmê, phronêsis, sophia, and nous. These virtues distinguish between Knowledge and wisdom and between the theoretical and the practical. Epistêmê (theoretical Knowledge) and technê (practical Knowledge) are familiar to us as science and technology, respectively (14). Phronêsis (practical wisdom) describes rational thinking capability that accounts for context and contingent facts, thus taking in "the particular." One places value on information that may change with events. Aristotle considered phronêsis the first of the four cardinal virtues because ethics guides the individual to place the community's good ahead of the individual's good. Phronesis is acquired through practice and observation: practice creates the experience while observing elders who model this virtue leads one to phronesis (15). Providence of foresight is the source for our word "prudence"; the Romans translated phronesis as "prudence." Phronesis is now more commonly translated as "practical wisdom."

These two meanings of art overlap through observation, which is all we have when we identify the exigent discrepancy or disruption (9). We find meaning in what we see, which we communicate to another person. This concept is an art as a practice and as a representation of the world. Techniques from the visual arts (negative space), literary arts (narrative, mystery, or suspense stories), and performing arts (story versus scene) can better inform our descriptions and communications.

\section{"Is there practicality in describing neonatology's art or practice through aesthetics, contextualization, affect, virtue, or observation? Dispassionate scientific theory guides diagnosis and treatment."}

Is there practicality in describing neonatology's art or practice through aesthetics, contextualization, affect, virtue, or observation? Dispassionate scientific theory guides diagnosis and treatment. Scientific rationality ensures scientific theory's integrity by isolating theory from practice and context $(16,17)$. But neonatologists operate through contextual relations entwined with people and work, building into routine operations their own logic of practice (16). This gap between theory and practice cannot be closed by merely borrowing a little from each. Engagement brings theory into the practical world and can close the gap (17); there is little discussion on how to engage or what drives engagement.

The Art of Neonatology emerges when practical engagement combines the science and practice of medicine. This coupling is more than taking a little from each as circumstances arise. In 1951 after 30 years of attempts, the science of high altitude climbing and the practice of high altitude climbing were each sufficiently developed to reach the summit of Mount Everest. But neither the domain of science or climbing could individually or in combination support a successful summit climb. Instead, it was a climbing scientist, Gifford Pugh, who melded the two into a single entity that enabled climbers to gain altitude (8).

Affect, rather than cognition, drives engagement, and it is contextual engagement that generates learning through practice (5). An essential quality of the physician is interest in humanity (2), the virtue of practical wisdom. "The secret of the care of the patient is 
in caring for the patient (2). Through the art of climbing, mountaineers gained altitude. Through the Art of Neonatology, neonatologists care for their patients and gain life.

\section{"Through the Art of Neonatology, neonatologists care for their patients and gain life."}

\section{Art as a representation of the world}

Initially, observation is all we have. Our engagement of a situation starts with our first look when we let our looking teach us. Collaboration in a situation begins with accurate and meaningful description. With experience, we can gain the "art of describing in darkness and through the fog." These are the visual arts.

\section{Visual Arts: Art as a rendering of the world}

\section{To see the detail: Prehistoric art and Muybridge}

Accurate observation and the rendering of action may reflect close and continued contact between the action and the artist observer. For example, cave paintings by upper paleolithic humans depicted walking quadrupeds with greater accuracy than paintings by modern artists. Not until Eadweard Muybridge's serial photographs of walking quadrupeds in the 1880s did contemporary artists depict quadruped walking with accuracy. Perhaps the more accurate observation and rendering by paleolithic humans came about because these were the animals they had intimate contact with (18). This realization demonstrates the possibility that an involved observer can accurately observe and communicate complex observations.

The skills of observation are portable. Louis Pasteur identified right-handed, left-handed, and symmetrical mirror-images of crystalline tartrates, marking the discovery of chemical enantiomers. The asymmetry of the facets is nearly unnoticeable. In his youth, Pasteur painted and drew, developing an eye for detail. Perhaps Pasteur's perception of detail, developed through his artwork, contributed to this identification (19).

A paramedic's poor documentation led him to meet alone with one of the authors (DvS), his EMS provider's medical director. Sensing resistance from the paramedic, the author asked about the paramedic's hobbies, surfing. The author inquired how the height of a shore wave is measured from the front or the back. The paramedic spontaneously presented a wave's characteristics - speed, height, momentum, how to get on and off the wave, etc. After he finished, the author asked why he couldn't describe a patient with that detail. He was met with silence. Several months later, the author received notification of an incident involving the paramedic. On scene at a traffic collision, three senior paramedics and the base hospital physician-approved transport of a patient to the local emergency department. The paramedic, junior to the others, called the physician to describe the patient. The physician upgraded the patient to a trauma response with transport to a trauma center where surgeons reduced a rapidly evolving tension pneumothorax.

\section{Perspective}

As a term in art, Linear perspective makes possible the depiction of three-dimensional objects in a two-dimensional space. This perspective allows for more abstract thinking about volumes and shapes without losing their concreteness, enabling mental calculation and communication. This abstraction is objective and guides information. Perspective as the psychological term, rather than the art term, refers to personal views and facts known to the individual who is subjective and influences perception.

The vanishing point in art marks the convergence of the lines that represent parallel lines in three-dimensional space. This convergence brings direction to the artwork in a similar manner; it can bring direction and coordination to the practice of medicine.

Negative Space

The environment carries information. Though the HRO engages the embedded problem (9), we cannot clearly identify the problem's boundaries or the environment. The environment carries information; the environment can direct meaning and relevance to different aspects of the problem. In this manner, the environment has a role similar to negative space in the visual arts.

\section{"Negative space is the space in a painting intentionally left empty to bring attention to the subject, the subject being the positive space."}

Negative space is the space in a painting intentionally left empty to bring attention to the subject, the subject being the positive space. If we were to look at a portrait with flowering bushes in the background, we would likely be unable to fully identify the painting's subject - the person or the flowers. Adding a shaft of sunlight or creating a clearing behind the portrait brings attention to the subject. In sculpture, negative space is the stone that is carved away to focus attention on the subject.

The use of negative space aids in describing and understanding situations. For example, if we look between the positive spacesthose parts that attract our attention-we see things we would have missed. In discussions with the authors, a plein-air artist uses negative space to shape the painting, and a hunter looks for horizontal lines of the prey species in the negative space between the vertical, horizontal space of the trees. This space does not refer to seeing the forest for the trees; it sees between the trees. Negative space forces a shift from the verbal left-side of the brain that processes familiarity to the brain's spatial right-side where novelty is processed.

We can see this effect when we draw a picture of something or someone right-side-up versus upside down. Because we cannot categorize when we use the upside-down approach, we often find that this kind of drawing produces a more accurate representation. We can also see the effect of negative space on comprehension when we draw a picture of our hand and then draw a separate picture of what we see between our fingers and around our hand. Drawing what appears around our hand gives a more 
accurate likeness (20).

The use of negative space as a description can aid staff in describing confusing or complex situations. Negative space describes what makes the situation important rather than stating what is not present. Describing a neonate by the amount of supplemental oxygen provided, the positive space, carries less information than providing the level of minimal titratable oxygen. The former tells us what the neonate is receiving; the latter tells us what is necessary for the neonate.

\section{"Perception may seem dependent on definitions, but definitions come up short in the turmoil of the liminal space. Descriptions of how something is used, an action performed, or a situation experienced all carry meaning."}

A group of respiratory care practitioners (RCP) in a subacute facility will call a consulting physician or intensivist early during the medical deterioration of a ventilator-dependent child. With little information available, they will use negative space to describe the child's condition and what treatments did not work. This representation gives meaning and interpretation to the positive space otherwise limited to physical examination and vital signs. This negative space presentation acts as negative feedback to ensure safety (21) or reciprocal feedback to modify plans (8). However, the RCPs report the frustration some physicians have when the physician is accustomed to ordering treatments, then waiting for the results before making further inquiry (personal communication to DvS).

Other examples of the use of negative space are descriptions of children as not small adults or premature neonates as not term infants. Rather than stating what they are, we state what they are not. Describing a child or premature neonate by negative space, such as the medical and environmental support necessary for incompletely developed or developing organs, can help the learner identify which previously learned information does not apply, building on the person's Knowledge.

\section{Color within context}

Painters use color beyond, ROYGBIV the constrained basic colors of the rainbow. For example, green is not a compromise between blue and yellow; it is a collaboration with varying amounts of each used depending on placement within the painting. Context chang-

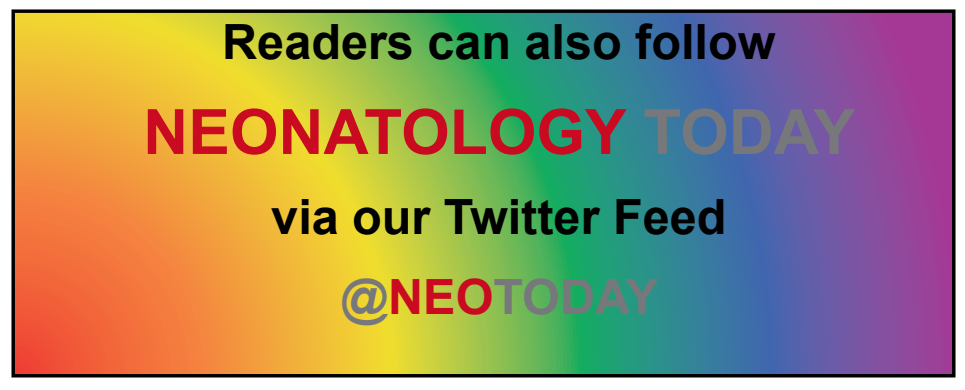

es the perceptions of color. For example, the same hue for yellowgreen leaves on a tree partially in front of a dark cloud changes the perception of the leaves when painted against darker clouds compared to a sunnier sky. This albedo effect can be noticed in a movie theater with a white screen. The projector cannot transmit black, yet night scenes show a dark sky on a white screen.

\section{Genres of painting}

The purpose of a painting may be to document, influence, or describe. One author (DvS) used various genres of artwork to help residents form their descriptions. Medical students and residents feel the essential tension between thorough and detailed presentations - too much and not enough but never right. To reduce this tension when presenting to the attending physician, the author offered different presentation styles based on some basic genres: Dutch still life, Hudson River School, Impressionism, and Cubism.

Dutch still-life paintings. These are known for having insects within the flowers. There are times we want an accurate and detailed presentation of a portion of the history and physical examination.

Hudson River School. These describe a grander but realistic contextual view of the patient yet retaining the details.

Impressionism. The artist uses brush strokes, rather than lines and contours, to provide an impression of the scene. If you happen to observe an artist painting an impressionist master in a museum, observe the number of brushstrokes the student and the master use. As the residents focus on giving an impression, their words ("brush strokes") become fewer yet carry more information. Contextualization of the patient to create a clinical picture, described by Peabody (2) "not just a photograph of a man sick in bed; it is an impressionistic painting of the patient surrounded by his home, his work, his relations, his friends, his joys, sorrows, hopes, and fears.".

Cubism. Minimalism can carry more information than we think. Geometric shapes depict the human form. When we look at a set of circles, triangles, and rectangles, we do not "see" a human, but we think of a human. When reporting disconfirming information to an intimidating attending, this method was favored by residents - they do not say the disconfirming information; they make the attending think of it.

Examining an infant admitted for apnea in the Emergency Department after hospital discharge that morning, one author (DvS) obtained a history of apnea after feeding and observed Sandifer Syndrome. The residents were hesitant to re-admit the child with the diagnosis of reflux because the discharging attending had stated firmly that, following the results of a radiologic study that morning, there was no reflux. The author presented the infant's history as a Dutch Still Life, an Impressionist painting, and a "Picasso" Cubist painting. The residents chose to go with Picasso.

\section{Interpretation and art}

Methods of interpreting art help healthcare professionals receive information from the patient, environment, and each other. The critical interpretation of art follows describing, analyzing, interpreting, and judging. The viewer wants to perceive and find meaning, and the artist wants to help. When we cannot make sense of the artwork, we adjust our understanding while the artist makes 
known the aesthetic intention (22). Interpretation, perception, and meaning work both ways between artist and viewer. This interaction parallels communications from individuals within a liminal event and the attending some distance from the event (11). The team within the event and the attending work together to ensure reliable and accurate information flow.

We can readily identify the utility of Barrett's (22) definitions:

Description of the object is without value judgments, analysis, or interpretation; answering the question, "What do you see?"

Analysis determines what the features suggest, deciding why the artist used certain features to convey specific ideas; answering the question, "How did the artist do it?" Interpretation establishes the broader context for this type of art, answering the question, "Why did the artist create it, and what does it mean?"

Judgment of work means giving it rank to other works and its originality, answering the question "Is it a good artwork?"

One author (DvS) routinely describes the various roles an individual has in communication (23): Identify, interpret, and translate. The individual identifies the salient aspects of the problem, interprets their relevance, and translates to give meaning to specific participants. During a resuscitation, information is translated to give meaning to the roles other team members have.

\section{"The individual identifies the salient aspects of the problem, interprets their relevance, and translates to give meaning to specific participants. During a resuscitation, information is translated to give meaning to the roles other team members have."}

Barrett (22) recognizes the multiple interpretations for artwork yet without the goal to arrive at a single interpretation. This focus is the purpose of translation but also the benefit of a "requisite diversity" of views. Interpretation is not specific, whereas translation is specific to the receiver. Constraining the range of possible interpretations increases the efficiency of information flow. The leader contributes by giving meaning to the interpretation, guiding the team's psychological response toward allostasis $(11,24)$. Interpretations will seldom be fully correct, but they can be reasonable and informative.

\section{Descriptions}

When one of the authors (DvS) became a paramedic, the Los Angeles County program was about six-years-old. Ambulance attendants and firefighters were unaccustomed to the use of medical terminology. Emergency physicians were not familiar with the equipment and abilities of paramedics. This inability to communicate contributed to such teaching moments as not telling the phy- sician the patient was "under arrest" as that statement would be followed with drugs for cardiac arrest. "The patient is in custody" became more readily used by a paramedic after a single event. Testing for the Babinski Reflex led to lessons learned on both sides of the radio conversation after an unconscious patient's audiotape was widely circulated in training classes. The radio nurse asked the paramedic if he had tried the Babinski. In the background, the paramedic is heard yelling, "Babinski! Babinski!" followed by "No, he doesn't respond to Babinski, either."

These problems led Ron Stewart, the physician in charge of paramedic training, to focus on accurate medical descriptions rather than medical terminology and included the patient's immediate environment. The goal was for the physician to understand the conditions of the patient and scene. More significantly, when paramedics received a questionable order, they would change their description of the patient to become more accurate or include the missing information. Persuasion or any type of manipulation in the description of the patient was not allowed. The paramedics also would precisely explain what equipment and drugs they had on hand relevant to the call. After hundreds of radio contacts with physicians, this became routine in medical care for the author (DvS).

\section{"The goal was for the physician to understand the conditions of the patient and scene. More significantly, when paramedics received a questionable order, they would change their description of the patient to become more accurate or include the missing information."}

Early in his ambulance career, before paramedics responded to "heart attack" calls, one author (DvS) learned to identify the "look of death" in patients with myocardial infarction. Though the patient would be talking to the family in the home, the ambulance attendants knew the patient would die on the way to the hospital. The author taught this to paramedics in local programs. During After Action interviews following a terrorist shooting, several paramedics mentioned they used that exact approach for part of their triage of shooting victims (25).

I keep coming back to "the look of death." Sensemaking seems too abstract and too distant to "describe" that engaged description. It is imminent in the EMS world of practice, part of an EMT's being-in-the-world, a world in which his pragmatic frame gives meaning to a patient vacillating between covert and overt decompensation. You're describing something other than a name or a concept; you're describing a look, a visual sense derived from experience. I could look at the same scene and see "nothing" that you see, yet see everything that my ethnographer experience "discloses."

This is powerful! I hope people spend time thinking about it. 
Karl Weick (personal communication)

\section{Art, Chaos, and Complexity}

\section{Art genres / styles}

The methods art historians use to classify painting genres can enhance our visual evaluation of complex and chaotic situations.

\section{"Mathematical measurements of borders and edges that reflect structure produced the degree of complexity. Measurements of diffuse fuzziness that reflected randomness gave a measure of entropy. Patterns of entropy produced an order- disorder spectrum."}

For example, the use of measures for entropy and complexity to evaluate an extensive series of digitalized paintings generated the same groupings as the classifications used by art historians (26). Mathematical measurements of borders and edges that reflect structure produced the degree of complexity. Measurements of diffuse fuzziness that reflected randomness gave a measure of entropy. Patterns of entropy produced an order-disorder spectrum. Patterns of complexity produced a simplicity-complexity spectrum. For standardization of art genres, the authors used the existing systems developed by Alois Riegl and Heinrich Wolfflin. Riegl used how the artist represented objects and Wolfflin how the artist used outlines and contours. Their artwork sample utilized online digitalized artworks of almost 140,000 paintings between the years 1031 and 2016.

Borders and edges (structure). Images formed by distinct and outlined parts yield many repetitions of a few ordinal patterns.

Diffuse fuzziness (randomness). Images composed of interrelated parts delimited by smudged edges produce a more random pattern.

Riegl distinguishes between artworks depicting tangible discrete objects ("haptic") versus objects interrelating in deep space using light, color, and shadow ("optic"). The former presents discrete objects, while the latter creates an open spatial continuum.

Wolfflin distinguishes between artworks containing objects with discrete borders and clearly outlined shapes ("linear") versus fuzzy outlines and contours that are subtle, smudged boundaries that merge images to give the idea of fluidity.

The authors (26) use mathematical equations to evaluate art genres can help us better understand the concepts of chaos and complexity. "Permutation entropy" as a measure between orderdisorder and "statistical complexity" for simplicity-complexity can help us better characterize the unexpected.
Often, we hear people say, "We are ready for anything." They understand entropy and believe there is a limit to the entropy change in their NICU. Permutation entropy measures the system's freedom to form any sequence of permutations without a change in the maximum entropy of the system (27). The care of the critically ill neonate may be finite, but the permutations of that care have their own entropy and can change in unexpected sequences or progressions.

With some almost helpless resignation, people will bemoan a "random" event, or the system has become too complex. Statistical complexity measures the complexity of the system's structure independent of random processes (28). It is worth recognizing there are independent contributions to system complexity that come from the randomness that is distinct from the system's structural complexity.

These measures distinguished between classifications of artworks and help us observe and appreciate the turmoil at the beginning of a resuscitation.

\section{"Complexity and entropy are measures of information contained within the painting or system. Entropy is a time-based measure of divergence as the system moves from initial conditions toward randomness."}

Complexity and entropy are measures of information contained within the painting or system. Entropy is a time-based measure of divergence as the system moves from initial conditions toward randomness. Chaos is also time-based, but it occurs at a threshold reaction rate (29). Complexity has a large number of elements, but only a few have linear interactions. Chaos has only a few elements, but they have nonlinear interactions.

Information as Shannon Information Entropy is created from the conversion of uncertainty to certainty. The certainty of ordered systems means they do not contain information. e do not learn anything new from an ordered system. Disordered systems, on the other hand, generate information as they become ordered from the conversion of uncertainty (disorder) to certainty (order) (30). The complexity develops from the interaction of entropy (disorder) and disequilibrium; therefore, complexity also carries information (27). Regular, ordered paintings such as Minimalism paintings have small entropy values and carry little information, while paintings with less regularity, such as Pollock's drip painting, have larger entropy values and carry more information.

The genres of art also map to the genres of education and medicine. Romanticism rigidly distinguishes objects separated by flat surfaces. The low complexity and decreased randomness are ideal for documentation and introductory programs. The looser, smudged brushstrokes of Impressionism avoid the creation of sharp edges to show complexity, depth, light, and shadow, while the merged images and smudged boundaries reveal the complexity of life. In the words of Peabody (2), "an impressionistic painting 
of the patient surrounded by his home, his work, his relations, his friends, his joys, sorrows, hopes, and fears."

Modern and Postmodern Art's transition gave artwork clear outlined shapes that depict tangible discrete objects having less complexity and randomness. This imaging is good for a straightforward evaluation of a stable neonate. It would be of less use at the beginning of abrupt patient deterioration.

\section{"Our digression to understanding chaos} and complexity through art has a serious purpose. An extensive literature in medicine and emergency care discusses chaos and complexity, the causes, identification, and necessary responses."

Our digression to understanding chaos and complexity through art has a serious purpose. An extensive literature in medicine and emergency care discusses chaos and complexity, the causes, identification, and necessary responses. However, in practice, we encounter exigent situations somewhere between the covert compensated state and the overt decompensated state (9). We engage in saving a life. Determining uncertainty, complexity, or chaos detract from our primary objective. What we have readily available is observation. The above exercise guides us to identify borders of instability, edges that differentiate, and fuzzy, unclear elements. We engage areas without structure and evaluate regions of randomness. We reduce complexity and act from reciprocal feedback. There is time enough to tell outsiders if it was uncertainty, complexity, or chaos.

\section{Observation and Inductive Processes}

During the first moments of resuscitation, it matters little whether we have uncertainty, complexity, or chaos. All we have is observation. Only observation will lead us to the Knowledge necessary to engage the emergency. "The kind of knowledge which is supported only by observations and is not yet proved must be carefully distinguished from the truth," Leonhard Euler (31). Distinguishing false Knowledge from the truth, solely by observation, can engender fear of making a mistake. Such generalized fear will cause greater harm than the act or mistake itself (32). Acting on imperfect Knowledge is not a mistake or error. Actions become mistaken when circumstances change or new information becomes available, but the action does not become an error until its completion (33).

Engagement describes continuous interaction with a situation until resolution, obviating the effects of error. In this approach, errors serve to identify a change in circumstances, correct heuristic biases, and mark the limits of individual capabilities or operational performance (21). Identification of an error initiates learning as the environment begins to correct our beliefs or ideas (31). Viewed from this frame of reference may make it difficult to recognize the induction process introduced by Euler within this quote above. Observation, then, begins the inductive process. "We should use such a discovery as an opportunity to investigate more than exactly the properties discovered and to prove or disprove them; in both cases, we may learn something useful," Leonhard Euler (31).

In the HRO, engagement bridges the gap between theory and practice during the constant action of a crisis (9). The engaged individual is continuously thinking and making judgments, using those judgments for the improvisation that directs self-organization (25) (34). This engagement better describes the results of the more accurate translation of René Descartes' dictum, cogito ergo sum, "I am thinking; therefore I exist" (34).

When the environment carries information, one is constantly thinking. To stop thinking is a mistake. When premature diagnosis terminates inquiry, or members do not take action to avoid error, there develops the more insidious "error from not acting" (35). These errors cannot easily be detected, becoming organizational Knowledge enforced by moral authority.

In a back-and-forth discussion with Karl Weick regarding cognitive dissonance as a risk for uncorrected errors and failures, Weick concluded with this reply [his comments in italics]:

On page 8 of chapter 2 of one of your manuscripts, the chapter is called Logic of Operations, you [DvS] say this: "Knowledge in the threatening, unstructured state takes a different form from what we are accustomed to. Knowledge acts as a degree of belief that must be updated from information generated during the event. Mistaken beliefs must be identified and corrected, no matter how dearly held. Compared to an updated belief, a mistaken belief may only depend on its presence at initiation or the length of time it is held. Events happen continuously, creating the need for dynamic reasoning processes and easier acceptance of new, disconfirming evidence. Longheld entrusted beliefs must be freely questioned, not an easy thing to do for most people, regardless of the level of skill or logic used" (23).

The clash between a mistaken old belief and an updated belief would seem to be a form of dissonance. An interesting possibility is that the more you engage in dynamic reasoning, the less chance there is for dissonance between old belief and updated belief to develop and the fewer errors you make.

Karl Weick, personal communication

An inductive attitude is protective from error. The individual adapts to experience as efficiently as possible. "It requires a ready ascent from observation to generalizations and a ready descent from the highest generalizations to the most concrete observations. It requires saying "maybe" and "perhaps" in a thousand different shades (31).

It requires:

1. Intellectual courage, ready to revise any one of our beliefs;

2. Intellectual honesty, change a belief when there is a compelling reason to change it; and

3. Wise restraint, to not change a belief wantonly, without some good reason 
Inductive processes give us a chance to correct our ideas when they are wrong, to adapt them to reality.

George Pólya.(31)

"Engagement and an inductive attitude readily turn error away from harm and failure toward learning and serendipity. After all, how do we convert an accident into a fortunate discovery except by continued engagement and learning?"

Engagement and an inductive attitude readily turn error away from harm and failure toward learning and serendipity. After all, how do we convert an accident into a fortunate discovery except by continued engagement and learning? We draw upon our unrelated experiences and Knowledge, such as an artist's eye for detail and crystalline tartrates. Louis Pasteur "presented himself as a practitioner of the inductive scientific method, working outside of theories," creatively seizing opportunities that appeared accidental (36). His explanation:

"Dans les champs de l'observation, le hazard ne favorise que les espirits préparés."

(Where observation is concerned, chance favors only the prepared mind.)

\section{Conclusion}

Not by leaders, nor by rules, nor by design does the individual engage in a turbulent scene. The individual must have "the kind of knowledge which is supported only by observations and is not yet proved," Euler (31). One can confirm through observation, aligning experience with science for a sense of security and stability. Not to act is not to err, unintentionally and paradoxically adding to organizational Knowledge. With experience, one can develop a practice of neonatology. But confirming what is known is not reliable for the particular. Failure by not acting is not visible, errors cannot be corrected, and judgment cannot become refined.

The Art of Neonatology is the art observation. Let your looking teach you. Even in complexity and chaos, we observe elements that form structure. Thinking is continuous, "I am thinking; therefore I exist," René Descartes. We continuously exist in our environment with combined mind, emotions, and judgments, John Dewey's aesthetics of pragmatism. With continuous actions, some of our acts become mistakes. Our acts are not mistakes; they become mistaken from a change in circumstances, Marianne Paget. Whether we prove or disprove our mistakes, this kind of Knowledge is gained by induction, "we may learn something useful," Leonhard Euler. It is induction that gives us a chance to correct our ideas and adapt to reality, George Pólya. Inductive processes with involved inquiry generate "learning through observation." Louis Pasteur advised us, "Where observation is concerned, chance favors only the prepared mind."
In the final analysis, it is the individual who alone faces the turbulence. That person must enter the void supported solely by their ability to observe and act. We achieve high reliability through individuals such as these. The aesthetic makes this an art, the interest in humanity, as Francis Peabody (2) stated, "the secret of the care of the patient is in caring for the patient."

\section{"The art of a discipline is synthesis. Synthesis extends Knowledge to new heights. Alpine climbers gained height through the art of climbing. Neonatologists gain life through the Art of Neonatology."}

The art of a discipline is synthesis. Synthesis extends Knowledge to new heights. Alpine climbers gained height through the art of climbing. Neonatologists gain life through the Art of Neonatology. An individual can master the "science of neonatology." Perhaps an individual can approach mastery of the "practice of neonatology." But the Art of Neonatology, like High-Reliability Organizing, cannot be mastered. Mastery is an inductive process with continuous learning, continuous synthesis, continuous observation. Like Aristotle's practical wisdom, mastery is an art.

It took me four years to paint like Raphael, but a lifetime to paint like a child.

Pablo Picasso

\section{References:}

1. Beardsley M. An aesthetic definition of art. In: Curtler H, editor. What is art New York, NY: Have Publications; 1983.

2. Peabody FW. The care of the patient. The Journal of the American Medical Association. 1927;88(12):877-82.

3. O'Sullivan S. The aesthetics of affect: Thinking art beyond representation. Angelaki: journal of theoretical humanities. 2001;6(3):125-35.

4. Adajian T. The Definition of Art. In: Zalta EN, editor. The Stanford Encyclopedia of Philosophy Stanford University; 2018.

5. Leddy T. Dewey's Aesthetics. In: Zalta EN, editor. The Stanford Encyclopedia of Philosophy: Stanford University; 2020.

6. Spradley JP. Participant observation. Long Grove, IL: Waveland Press; 2016.

7. Aristotle. Nicomachean Ethics. Chicago, IL: The University of Chicago Press; 2011.

8. van Stralen D, Mercer TA. High Altitude Climbing, High Reliability, COVID-19, and the Power of Observation. Neonatology Today. 2021;16(1):68-79.

9. van Stralen D. Pragmatic HRO during Pandemic COVID-19. Neonatology Today. 2020;15(4):3-9.

10. Mack JE, Hickler H. Vivienne: The Life and Suicide of an Adolescent Girl. Boston, MA: Little, Brown and Company 1981.

11. van Stralen D, Mercer TA. Pragmatic High-Reliability Organizations (HRO) Modulate the Functions of Stress and Fear Behaviors During Pandemic COVID-19: The Stress-FearThreat Cascade. Neonatology Today. 2020;15(10):126-34. 
12. Arnsten AFT. Stress signalling pathways that impair prefrontal cortex structure and function." Nature Reviews Neuroscience. 2009;10(6):410-22.

13. van Stralen D, Mercer TA. During Pandemic COVID-19, the High-Reliability Organization (HRO) Identifies Maladaptive Stress Behaviors: The Stress-Fear-Threat Cascade. Neonatology Today. 2020;15(11):113-24.

14. Parry R. Episteme and Techne. In: Zalta EN, editor. The Stanford Encyclopedia of Philosophy Stanford University; 2014.

15. Ryan S. Wisdom. In: Zalta EN, editor. The Stanford Encyclopedia of Philosophy Stanford University; 2014.

16. Sandberg J, Tsoukas H. Grasping the logic of practice: Theorizing through practical rationality. Academy of Management Review. 2011;36(2):338-60.

17. Zundel $M$, Kokkalis $P$. Theorizing as engaged practice. Organization Studies. 2010;31(9-10):1209-27.

18. Horvath G, Farkas E, Boncz I, Blaho M, Kriska G. Cavemen were better at depicting quadruped walking than modern artists: erroneous walking illustrations in the fine arts from prehistory to today. PloS one 2012;7(12):e49786.

19. Root-Bernstein RS. Visual thinking: The art of imagining reality. Transactions of the American Philosophical Society 1985;75(6):50-67.

20. Edwards $B$. The new drawing on the right side of the brain: The 1999, 3rd Edition. New York, NY Penguin Publishing; 1999.

21. van Stralen D, Gambino W. Error as a Faulty Failure Signal. Neonatology Today. 2020;15(9):114-7.

22. Barrett T. Criticizing Art: Understanding the Contemporary. Mountain View, California: Mayfield Publishing Company; 1994.

23. van Stralen D, Byrum SL, Inozu B. High Reliability for a Highly Unreliable World: Preparing for Code Blue through Daily Operations in Healthcare: CreateSpace Independent Publishing Platform; 2017.

24. van Stralen D, Mercer TA. Flight Decks and Isolettes: HighReliability Organizing (HRO) as Pragmatic Leadership Principles during Pandemic COVID-19. Neonatology Today. 2020;15(7):113-22.

25. van Stralen D, McKay S, Williams GT, Mercer TA. Tactical Improvisation: After-Action/ Comprehensive Analysis of the Active Shooter Incident Response by the San Bernardino City Fire Department December 2, 2015. San Bernardino, CA: San Bernardino County Fire Protection District; 2017.

26. Sigaki HY, Perc M, Ribeiro HV. History of art paintings through the lens of entropy and complexity. Proceedings of the National Academy of Sciences 2018;115(37):E8585E94.

27. Bandt C, Pompe B. Permutation entropy: a natural complexity measure for time series. Physical review letters 2002;88(17).

28. Feldman DP, Crutchfield JP. Measures of statistical complexity: Why? Physics Letters A. 1998;238(4-5):244-52.

29. May RM. Biological populations with nonoverlapping generations: stable points, stable cycles, and chaos. Science. 1974;186(4164):645-7.

30. van Stralen D. Ambiguity. Journal of Contingencies and Crisis Management. 2015;2(23):47-53.

31. Pólya G. Mathematics and plausible reasoning: Induction and analogy in mathematics. Princeton, NJ: Princeton Uni- versity Press; 1954.

32. van Stralen D, Mercer TA. Pandemic COVID-19, the HighReliability Organization (HRO), and the Ecology of Fear. Neonatology Today. 2020;15(12):129-38.

33. Paget MA. The unity of mistakes: A phenomenological interpretation of medical work. Philadelphia, PA: Temple University Press; 1988.

34. Gaukroger S. Descartes: An intellectual biography. Oxford, UK: Clarendon Press; 1995.

35. Weick KE. Sensemaking in Organizations. Thousand Oaks: CA: Sage Publications; 1995.

36. Geison GL. The private science of Louis Pasteur. Princeton, NJ: Princeton University Press; 2014.

Disclosure: The authors have no disclosures.
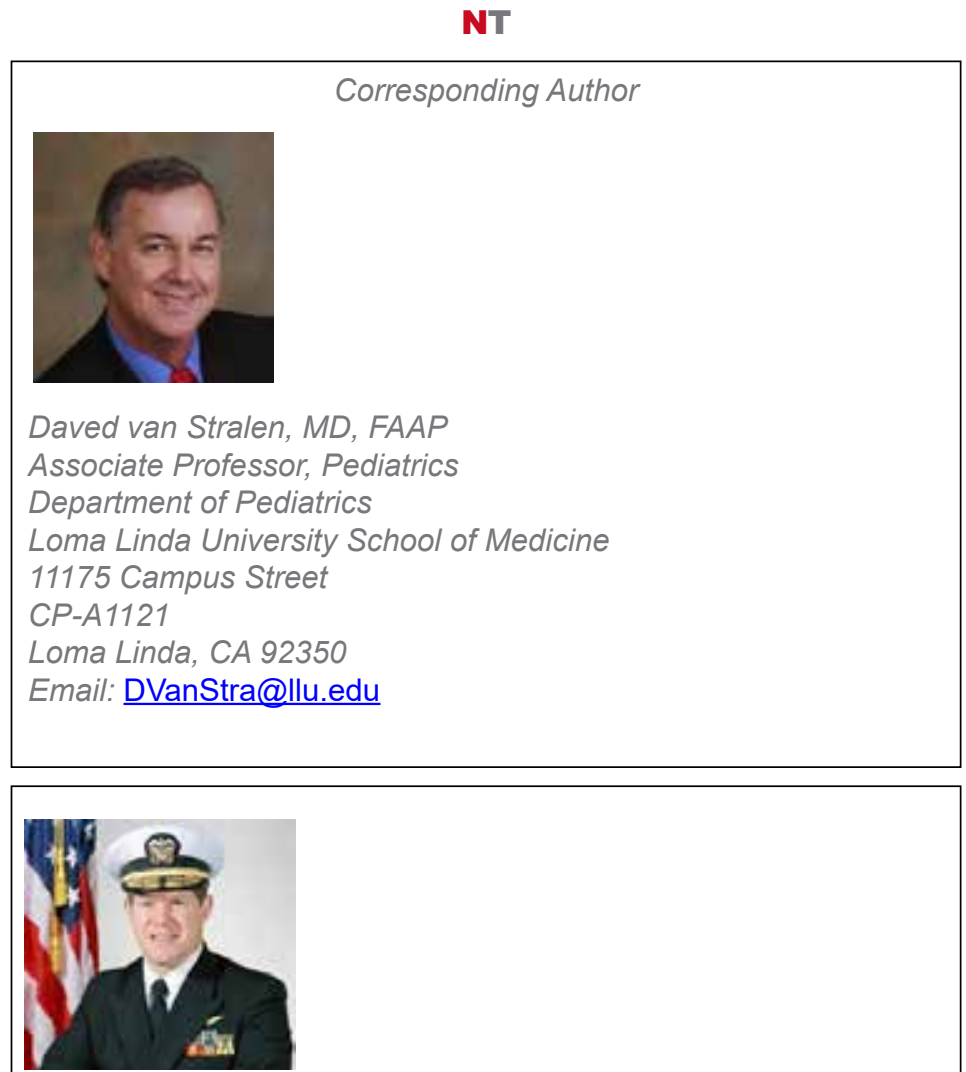

Thomas A. Mercer

Rear Admiral

United States Navy (Retired)

Readers can also follow NEONATOLOGY

via our Twitter Feed

@NEOTO 


\section{Acknowledgments}

Raymond Novaco, Professor, Psychology and Social Behavior, School of Social Ecology, University of California, Irvine, California

Karl Weick- review and editing, Rensis Likert Distinguished University Professor of Organizational Behavior and Psychology, Emeritus, University of Michigan

William J. Corr, formerly with the Los Angeles City Fire Department, now deceased

Errol van Stralen, Ancora Education

Sean D. McKay, Element Rescue, LLC

William Gambino, CIV, DoD

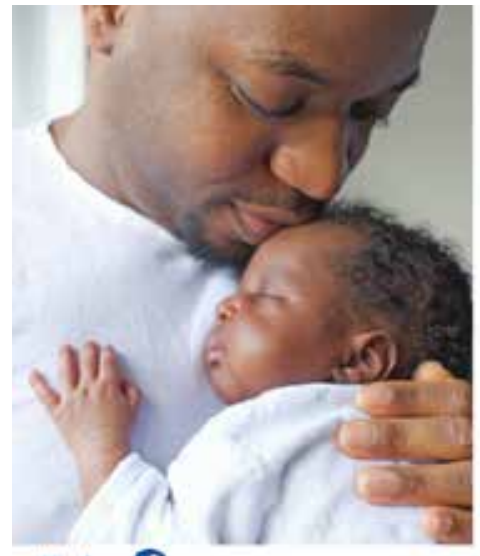

Did you know that

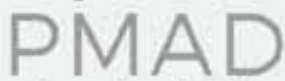

related suicides

account for

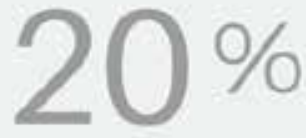

of Postpartum

Maternal Deaths?

Join O NPA

nationalperinatal_org/mental_health

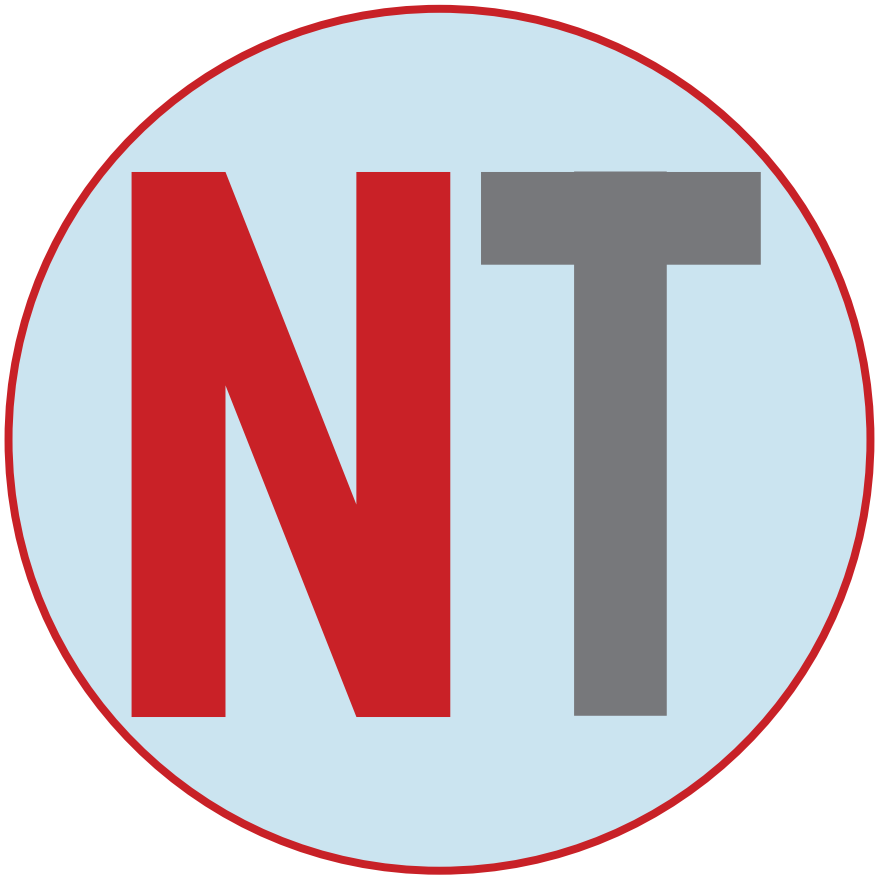

FREE RESOURCES FOR YOUR NICU

\section{Coping During COVID-19

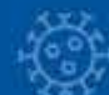

sits

Targeted interventions to improve the mental health of parents, infants, families, and providers

\section{BONDING WITH YOUR BABY}
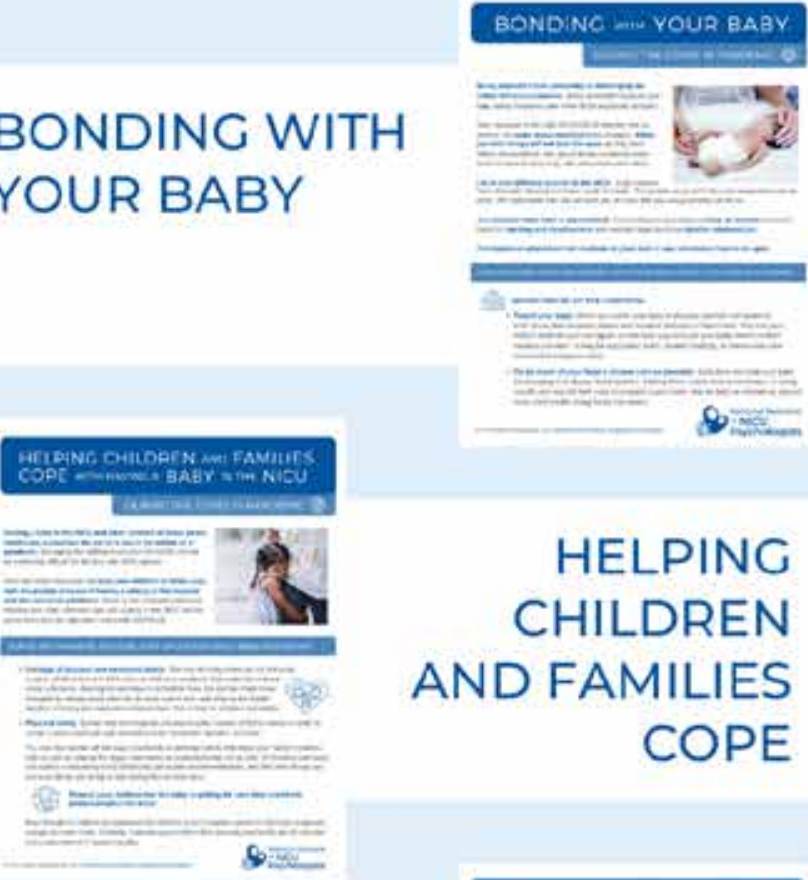

\section{HELPING CHILDREN AND FAMILIES COPE}

CAREGIVERS NEED CARE TOO

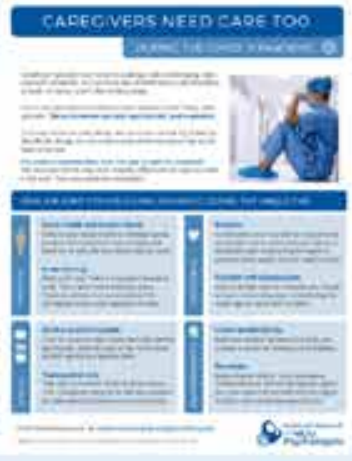

